\title{
Association Between the Alterations in the Blood Routine Check Parameters and Cognitive Impairment in the Shenzhen Ageing-related Disorder Cohort in China
}

\section{Kaiyu Wu}

Jilin University First Hospital

\section{Chunyan Xu}

Shenzhen University College of Medicine

Xiaoping Tian

Shenzhen University College of Medicine

\section{Guozhen Qiu}

Shenzhen University College of Medicine

Wei Liu

Shenzhen Center for Disease Control and Prevention

\section{Qiwen Guo}

Shenzhen University College of Medicine

\section{Xueya Meng}

Shenzhen University College of Medicine

\section{Chunchun Chen}

Shenzhen University College of Medicine

Jianjun Liu

Shenzhen Center for Disease Control and Prevention

\section{Zhiwei Guo}

Shenzhen Luohu Hospital Group Luohu People's Hospital

\section{Haifei Chen}

Shenzhen Luohu Hospital Group Luohu People's Hospital

\section{Kangding Liu}

Jilin University First Hospital

Feiqi Zhu ( $\sim$ zfqzsu2004@aliyun.com )

Shenzhen University College of Medicine https://orcid.org/0000-0003-4704-8928

\section{Research}


Keywords: Cognitive impairment, blood parameters, lymphocyte to monocyte ratio, packed-cell volume, platelet count, monocyte count, mean corpuscular hemoglobin concentration

Posted Date: August 13th, 2021

DOl: https://doi.org/10.21203/rs.3.rs-796144/v1

License: (c) (1) This work is licensed under a Creative Commons Attribution 4.0 International License. Read Full License 


\section{Abstract}

Background: Previous studies have suggested a correlation between alterations in the routine blood check markers and cognitive impairment.

Objective: We aimed to explore the relationship between routine blood parameters and cognitive impairment.

Methods: In this retrospective case-control study, 8,025 people over 60 years of age were enrolled from 51 community health centers in Luohu District of Shenzhen City from 2017 to 2018 . According to the minimental state examination (MMSE) score and educational level, participants were divided into a cognitive impairment group ( $n=385)$ and normal cognitive function group $(n=7,640)$. Nonparametric test, chi-square test, and binary logistic regression were applied to analyze the data.

Results: Compared to the normal cognitive function group, the lymphocyte-to-monocyte ratio (LMR), packed-cell volume (PCV), and platelet count (PLT) in the cognitive impairment group were significantly lower (7.26 [5.50,8.81] vs. $7.45[5.80,9.35] ; 40.1[36.65,42.95]$ vs. $40.5[37.7,43.1] ; 216[190,258]$ vs. 228 $[196,261], p<0.05$, respectively), while the monocyte count and mean corpuscular hemoglobin concentration $(\mathrm{MCHC})$ were higher $(0.31[0.25,0.38]$ vs. $0.3[0.24,0.37] ; 336[330,343]$ vs. 335 [328, 342], $p<0.05$, respectively). Additionally, $\mathrm{MCHC}$ [odds ratio (OR):1.011, 95\% confidence interval (Cl) :1.001$1.021, p=0.033$ ] and PCV [OR: $0.993,95 \% \mathrm{Cl}: 0.987-0.999, p=0.033)$ were independently associated with cognitive impairment.

Conclusion: The alterations in some routine blood check parameters, including reduced LMR, PCV, and PLT, and elevated monocyte count and MCHC, may be associated with cognitive impairment.

\section{Background}

Dementia is becoming increasingly common in the aging population. Alzheimer's disease (AD), the principal type of dementia, has a devastating impact on human health and the economy worldwide [1]. Over the past decades, many scientific experiments have been conducted to explore the pathology of $A D$ and to discover novel treatments. $A D$ is characterized by neuronal and synaptic degeneration, together with an extensive number of amyloid-beta $(A \beta)$ plaques and neurofibrillary tangles, which may occur before the appearance of symptoms [2]. Screening for reliable and suitable biomarkers that can be used before the clinical manifestations become apparent has been an urgent issue for $A D$ investigators. Compared to cerebrospinal fluid, which is now widely used as a diagnostic source to aid the clinical diagnosis of $A D$, peripheral blood samples are easier to collect and comparatively less invasive. As highly invasive procedures are not appropriate for screening, accumulating evidence shows that besides the well-known markers, $A \beta$ and tau protein in blood, many routine peripheral blood parameters may be potentially useful as novel biomarkers to reflect the prognosis of $A D$ and other neurological disorders. However, results have been inconsistent and lack reproducibility [3-5]. It has been shown that the mean platelet volume (MPV) and platelet distribution width (PDW) are decreased in patients with mild cognitive 
impairment $(\mathrm{MCl})$ and $A D$. Lower hemoglobin levels are associated with cognitive impairment, and elevated red cell distribution width (RDW) is related to a reduced brain volume and cognitive dysfunction $[6,7]$. Here, we investigate further the association between alterations in the peripheral blood parameters and cognitive impairment among the elderly in Shenzhen City, Guangdong, China.

\section{Material And Method}

\section{Study population}

From 2017 to 2018, we enrolled 9,411 participants aged over 60 years old from 51 community health centers in Luohu District of the third affiliated hospital of Shenzhen Univerisity Medical College, Shenzhen City. A total of 8,025 participants was enrolled based on the following inclusion criteria: age $>60$ years, intact visual and auditory function, and ability to complete the mini-mental state examination (MMSE). Excluded were persons with acute or chronic infectious diseases, malignant tumors, hematologic system diseases, or severe liver and kidney diseases that may affect the routine blood indicators. Subjects with MMSE score $\leq 21$ (for subjects with an education level of primary school or below) or MMSE score $\leq 24$ (for subjects with an education level of secondary school and above) were considered to have cognitive impairment.

Participants were divided into a cognitive impairment group $(n=385)$ and a normal cognitive function group $(n=7640)$ (Fig. 1). Written informed consent was obtained, which included permission for the analysis and data sharing. This study was approved by the Independent Ethics Committee of the third affiliated hospital of Shenzhen University Medical College.

\section{Clinical variables}

The routine laboratory parameters were measured, including white blood cell (WBC), lymphocyte count, neutrophil count, monocyte count, lymphocyte\%, neutrophil\%, monocyte\%, red blood cell count (RBC), hemoglobin (HGB), mean corpuscular volume (MCV), mean corpuscular hemoglobin (MCH), mean corpuscular hemoglobin concentration (MCHC), packed-cell volume (PCV), platelet count (PLT), MPV, PDW, and RDW, consisting of red cell distribution width standard deviation (RDW-SD) and red cell distribution width coefficient variable (RDW-CV). The neutrophil-to-lymphocyte ratio (NLR), lymphocyte to monocyte ratio (LMR), and platelet-to-lymphocyte ratio (PLR) were also calculated.

\section{Statistical analysis}

Data obtained from participants in the cognitive impairment group and the normal cognitive function group were compared with respect to MMSE score, laboratory results, and including. The KolmogorovSmirnov test was used to assess the normality of continuous variances. Participants' characteristics were summarized as means (standard deviation) or medians (first quartile, third quartile) for continuous data (dependent on their distribution), and counts (proportions) for categorical variables. Continuous data were analyzed using the Mann-Whitney test if there was a skewed distribution. Categorical data were analyzed using the chi-square test. A binary logistic regression analysis was performed for statistically 
different indicators. All statistical analyses in this study were performed using SPSS software (version 26.0; IBM SPSS Inc., Chicago, IL, USA), and statistical significance was set at $p<0.05$.

\section{Results}

In total, 385 elderly participants with cognitive impairment (median age: 68 [64, 74] years, 203 women [52.70\%]) and 7,640 elderly participants with normal cognitive function (median age: $67(63,71)$ years, 4,408 women [57.70\%]) were included in this study. between the two groups.

Blood parameters were significantly different between the cognitive impairment group and normal cognitive function group (Table 1): $\operatorname{LMR}(7.26[5.50,8.81]$ vs. $7.45[5.80,9.35], p<0.05) ;$ PCV $(40.10$ $[36.65,42.95]$ vs. $40.50[37.70,43.10], p<0.05)$; $\operatorname{PLT}(216[190,258]$ vs. $228[196,261], p<0.05)$;

monocyte count $(0.31[0.25,0.38]$ vs. $0.30[0.24,0.37], p<0.05)$; and $\mathrm{MCHC}(336[330,343]$ vs. $335[328$, 342], $p<0.05$ ). The binary logistic regression analysis showed that $\mathrm{MCHC}$ (odds ratio [OR]:1.011, 95\% confidence interval [Cl] :1.001-1.021, $p=0.033]$ and PCV (OR: 0.993, 95\% Cl: 0.987-0.999, $p=0.033$ ) were independently associated with cognitive impairment (Table 2). 
Table 1

Demographics and laboratory parameters of the study population

\begin{tabular}{|c|c|c|c|}
\hline Parameters & $\begin{array}{l}\text { Cognitive impairment group } \\
(n=385)\end{array}$ & $\begin{array}{l}\text { Normal cognitive function group } \\
(n=7,640)\end{array}$ & $p$ value \\
\hline Age & $68(64,74)$ & $67(63,71)$ & $0.001^{*}$ \\
\hline $\begin{array}{l}\text { Sex } \\
\text { (female\%) }\end{array}$ & $203(52.70 \%)$ & $4408(57.70 \%)$ & 0.054 \\
\hline WBC & $6.54(5.69,7.46)$ & $6.41(5.53,7.43)$ & 0.242 \\
\hline LYMPH & $2.18(1.78,2.63)$ & $2.2(1.83,2.60)$ & 0.500 \\
\hline NEU & $3.72(3.11,4.34)$ & $3.65(3.01,4.42)$ & 0.282 \\
\hline MONO & $0.31(0.25,0.38)$ & $0.3(0.24,0.37)$ & $0.014 *$ \\
\hline LMR & $7.26(5.50,8.81)$ & 7.45(5.79,9.35) & $0.028^{*}$ \\
\hline NLR & $1.71(1.32,2.18)$ & $1.66(1.31,2.12)$ & 0.218 \\
\hline PLR & $101.92(82.86,126.65)$ & $103.37(84.66,126)$ & 0.292 \\
\hline Lymphocyte\% & $33.60(28.35,39.55)$ & $34.60(29.50,39.80)$ & 0.122 \\
\hline Neutrophil\% & $57.20(52.20,62.85)$ & $57.50(52,62.80)$ & 0.727 \\
\hline Monocyte\% & $4.70(3.90,5.70)$ & $4.60(3.80,5.50)$ & 0.067 \\
\hline RBC & $4.55(4.27,4.82)$ & $4.56(4.30,4.84)$ & 0.148 \\
\hline HGB & $137(130,147)$ & $138(130,146)$ & 0.701 \\
\hline $\mathrm{MCH}$ & $30.60(29.60,31.50)$ & $30.50(29.50,31.40)$ & 0.078 \\
\hline $\mathrm{MCHC}$ & $336(330,343)$ & $335(328,342)$ & $0.028^{*}$ \\
\hline MCV & $91.20(88.50,93.40)$ & $90.80(88.10,93.50)$ & 0.296 \\
\hline PCV & $40.10(36.65,42.95)$ & $40.50(37.70,43.10)$ & $0.034^{\star}$ \\
\hline PLT & $216(190,258)$ & $228(196,261)$ & $0.008^{*}$ \\
\hline MPV & $10.20(9.70,10.85)$ & $10.30(9.70,10.90)$ & 0.981 \\
\hline PDW & $11.60(10.55,12.90)$ & $11.60(10.60,12.90)$ & 0.855 \\
\hline
\end{tabular}

Abbreviations: WBC, white blood cell; LYMPH, lymphocyte count; NEU, neutrophil count; MONO, monocyte count; LMR, lymphocyte-to-monocyte ratio; NLR, neutrophil-to-lymphocyte ratio; PLR, platelet-to-lymphocyte ratio; $\mathrm{RBC}$, red blood cell; $\mathrm{HGB}$, hemoglobin; $\mathrm{MCH}$, mean corpuscular hemoglobin; $\mathrm{MCHC}$, mean corpuscular hemoglobin concentration; $\mathrm{MCV}$, mean corpuscular volume; PCV, packed-cell volume; PLT, platelet count; MPV, mean platelet volume; PDW, platelet distribution width; RDW-CV, red cell distribution width coefficient variable; RDW-SD, red cell distribution width standard deviation; *Statistically significant differences at $p<0.05$ (2-tailed). 


\begin{tabular}{|c|c|c|c|}
\hline Parameters & $\begin{array}{l}\text { Cognitive impairment group } \\
(n=385)\end{array}$ & $\begin{array}{l}\text { Normal cognitive function group } \\
(n=7,640)\end{array}$ & $p$ value \\
\hline RDW-CV & $13.20(12.80,13.70)$ & $13.20(12.70,13.70)$ & 0.907 \\
\hline RDW-SD & $42.80(41.10,44.65)$ & $42.60(41.00,44.30)$ & 0.192 \\
\hline \multicolumn{4}{|c|}{$\begin{array}{l}\text { Abbreviations: WBC, white blood cell; LYMPH, lymphocyte count; NEU, neutrophil count; MONO, } \\
\text { monocyte count; LMR, lymphocyte-to-monocyte ratio; NLR, neutrophil-to-lymphocyte ratio; PLR, } \\
\text { platelet-to-lymphocyte ratio; RBC, red blood cell; HGB, hemoglobin; MCH, mean corpuscular } \\
\text { hemoglobin; MCHC, mean corpuscular hemoglobin concentration; MCV, mean corpuscular volume; } \\
\text { PCV, packed-cell volume; PLT, platelet count; MPV, mean platelet volume; PDW, platelet distribution } \\
\text { width; RDW-CV, red cell distribution width coefficient variable; RDW-SD, red cell distribution width } \\
\text { standard deviation; *Statistically significant differences at } p<0.05 \text { (2-tailed). }\end{array}$} \\
\hline
\end{tabular}

Table 2

Associations of peripheral blood biomarkers with cognitive impairment

\begin{tabular}{|lcc|}
\hline Peripheral blood markers & Odds Ratio $(95 \% \mathrm{Cl})$ & $\boldsymbol{p}$ value \\
\hline MONO & $1.362(0.441,4.213)$ & 0.591 \\
\hline LMR & $0.993(0.944,1.043)$ & 0.767 \\
\hline MCHC & $1.011(1.001,1.021)$ & $0.033^{*}$ \\
\hline PCV & $0.993(0.987,0.999)$ & $0.033^{*}$ \\
\hline PLT & $0.998(0.996,1.000)$ & 0.070 \\
\hline $\begin{array}{l}\text { Abbreviations: Cl, confidence interval; MONO, monocyte count; LMR, lymphocyte-to-monocyte ratio; } \\
\text { MCHC, mean corpuscular hemoglobin concentration; PCV, packed-cell volume; PLT, platelet count; }\end{array}$ & \\
\hline *Statistically significant differences at $p<0.05$ (2-tailed). & \\
\hline
\end{tabular}

\section{Discussion}

We evaluated the association between alterations in routine blood parameters and cognitive impairment among 8,025 elderly patients. Decreased LMR, PCV, and PLT, and increased monocyte count and MCHC were associated with cognitive impairment among the elderly participants, and may be useful for the early evaluation and diagnosis of AD.

Platelets play important roles in neurodegenerative, cardiovascular, and psychiatric diseases, and have been widely used as a peripheral model to study the molecular mechanisms of $A D[8,9]$. Platelets are the primary source of circulating amyloid precursor protein (APP), and APP may represent the major source of $A \beta$ (the cleavage product of APP) detected in the whole blood [10]. It has been demonstrated that APP is expressed at similar levels in the platelets and brain [11]. Previous studies have suggested that platelet APP metabolism may promote $A \beta$ accumulation in the brain [12]. A recent study identified the essential functions of the APP family with regard to normal hippocampal function and circuits during development 
[13]. One study showed that the platelet level was statistically lower in patients with AD, which is consistent with our findings [9]. Some studies have also shown that platelets can accumulate and release neurotransmitters, such as serotonin, glutamate, and dopamine. Glutamate is a pivotal excitatory neurotransmitter in the central nervous system, which is related to cognitive function [14]. It has also been verified that the tau protein, another important pathophysiological component of $A D$, is present in platelets $[15,16]$. In addition, it has been shown that platelet size reflects platelet activity, which is related to a variety of proinflammatory diseases. MPV, the most commonly used measure of platelet size, is an early marker of activated platelets, and has different trends in different diseases [17]. PDW, another platelet index, can also indicate variations in the platelet size. Although some previous studies have indicated that MPV and PDW were decreased in $\mathrm{MCl}$ and $\mathrm{AD}$ patients $[18,19]$, we did not find significant differences in MPV and PDW levels between these two groups (Table 1).

In our study, there were significant differences in the monocyte count and LMR between the two groups (Table 1). The inflammatory response is known to be a key factor in the generation of $A \beta$ plaques and neurofibrillary tangles, which initially execute protective functions but eventually lead to neural degeneration and the manifestations of $A D$ [20]. Blood-derived leukocyte subpopulations, including lymphocytes, monocytes, and neutrophils, have been identified in the brains of patients with $A D$ and in corresponding animal models. Monocytes can reflect the innate immune inflammatory status, and chronic inflammation might cause an increase in the monocytes circulating in the blood. Subsequently, high monocyte levels may activate glial cells to secrete cytokines such as IL-1 and TNF- $a$ that damage the integrity of the blood-brain barrier (BBB), which is critical in the pathogenesis of AD [21]. Increasing evidence has shown that the serum levels of neopterin, which is produced by activated monocytes, are related to their capacity to release reactive oxygen species; these are higher in AD patients than in agematched healthy controls [22-24]. In line with several studies, decreased lymphocyte count in the cognitive impairment group was observed compared to the normal cognitive function group, although the difference was not significant in our study[4,21,25]. The decrease in the lymphocyte count, as part of the immune regulatory barrier, could reflect the body's stress response. TNF-a, released by microglia, is able to recruit lymphocytes from the peripheral circulation across the BBB into the central nervous system, which leads to a reduction in their numbers in the peripheral circulation. Furthermore, it has been shown that increased telomerase activity in lymphocytes can result in reduced lymphocyte proliferation in patients with $\operatorname{AD}[26,27]$. With regard to neutrophils, the increased neutrophil count is usually related to the occurrence, progression, and severity of inflammation. The depletion or inhibition of neutrophils reduces the pathogenesis and cognitive impairment of $A D$ in mouse models of cognitive dysfunction[28]. In recent years, an increasing number of studies have focused on NLR, a combined inflammatory biomarker that could have an advantage over a single leukocyte subtype and relatively higher clinical significance. The potential role of NLR in AD was first investigated by Kuyumcu et al., who found that NLR was significantly higher in AD patients compared to controls[29]. Several studies have also reported that elderly people with AD have a higher NLR than healthy controls, although we did not find a significant difference in the present study $[4,29,30]$. In summary, it was thought that the NLR was not specific enough to be used as a definitive diagnostic tool for $A D$ or $\mathrm{MCI}[20]$. 
According to our results, the PCV (Hct) level was decreased in the cognitive impairment group, which is consistent with a previous study [7]. PCV is viewed as a simple indicator of the number and volume of RBCs, which is an important indicator for diagnosing anemia. Although the erythrocyte count (4.55 vs 4.56) and hemoglobin level (137 vs 138) were not significantly different between the two groups, they were lower than those in the normal cognitive function group. $A D$ is a complex multifactorial disease, and anemia is known to be associated with AD. Studies have suggested that lower hemoglobin levels are associated with poor cognition [31, 32]. Several mechanisms might explain this phenomenon. Hemoglobin is a heme-containing protein that binds to oxygen, carbon monoxide, and nitric oxide. Erythrocytes are the most common location of hemoglobin. It is well known that the brain oxygen supply is mainly derived from the oxygen carried by RBCs. Numerous studies have revealed that exposure to hypoxia induces amyloidogenic processing of APP, leading to the accumulation of amyloid- $\beta$ peptides in the brain $[33,34]$. Notably, in AD, microglia defend the neurons by surrounding senile plaques to protect the brain and decrease damage. It has been demonstrated that hypoxia compromises the mitochondrial metabolism of microglia via hypoxia inducible factor 1 (HIF1) in AD [35]. One more possible explanation is that iron, an important component of hemoglobin which is involved in the transport of oxygen, accumulates in tissue stores and is not adequately mobilized in AD [36, 37]. Faux et al. found significantly greater prevalence of abnormally high serum ferritin together with lower plasma iron levels and transferrin saturation in AD, which is in line with studies that showed that iron metabolism was disrupted in cortical neurons in mouse models [7]. Additionally, studies have indicated that hemoglobin is a normal component of nerve cells, and plays a role in intraneural oxygen homeostasis. Moreover, decreased hemoglobin levels were associated with decreased cortical thickness in the frontal, temporal, parietal, and occipital regions, which are vital areas related to cognitive function [38]. Besides hemoglobin, it has been suggested that complement/complement receptor 1 (CR1) mediation is the predominant mechanism of $A \beta$ capture by erythrocytes [39].

We found that the $\mathrm{MCHC}$ level in the cognitive impairment group was higher than that in the normal group $(1.28[0.90,1.81]$ vs. $1.38[0.98,1.98], p=0.003)$, which is consistent with the results of a previous study [18]. MCHC indicates the amount of hemoglobin per unit volume, and correlates the hemoglobin content with the volume of the cell. In fact, $\mathrm{MCHC}$ levels have always been controversial among different studies, and several studies have demonstrated that decreased $\mathrm{MCHC}$ is related to cognitive impairment, while others have claimed that MCHC levels were not correlated with memory and cognitive abilities [40]. Therefore, it is difficult to explain the elevation of $\mathrm{MCHC}$ according to the current literature.

\section{Limitations}

First, it would be more accurate if we combined the MMSE with neuroimaging markers to evaluate cognitive function. Second, we did not rule out some chronic comorbidities, including hypertension, hyperlipidemia, and diabetes mellitus, which are important confounding factors that may affect blood cell profiles. Although we found that the differences in the blood parameters are associated with cognitive impairment, it remains unclear whether these changes are the cause or the result. 


\section{Conclusion}

Our study suggests that biomarkers in routine blood samples may be related to cognitive impairment, including a decrease in the LMR, PCV, and PLT, and elevation in the MONO and MCHC. Further studies are needed to investigate the pathophysiological and clinical significance of these changes in blood cells to offer new insights for the prevention and treatment of $A D$.

\section{Abbreviations}

MMSE: mini-mental state examination; LMR: lymphocyte-to-monocyte ratio; PCV: packed-cell volume; PLT: platelet count; MCHC: mean corpuscular hemoglobin concentration; AD: Alzheimer's disease; $A \beta$ : amyloid-beta; MPV: mean platelet volume; PDW: platelet distribution width; MCl: mild cognitive impairment; RDW: red cell distribution width; RBC: red blood cell count; HGB: hemoglobin; MCV: mean corpuscular volume; $\mathrm{MCH}$ : mean corpuscular hemoglobin; RDW-SD: red cell distribution width standard deviation; RDW-CV: red cell distribution width coefficient variable; NLR: neutrophil-to-lymphocyte ratio; PLR: platelet-to-lymphocyte ratio; APP: amyloid precursor protein; BBB: blood-brain barrier; HIF1: hypoxia inducible factor 1; CR1: complement/complement receptor 1.

\section{Declarations}

\section{Ethical Approval and Consent to participate}

All participating subjects signed the study's informed consent form that had also been approved by the Independent Ethics Committee of the third affiliated hospital of Shenzhen University Medical College.

\section{Consent for publication}

NA

\section{Availability of data and materials}

The data that support the findings of this study are available from the corresponding author, upon reasonable request.

\section{Competing interests}

The authors have no conflict of interest to report.

\section{Funding}

This work was supported by Key project of Shenzhen Science and Technology Commission (JCYJ20200109143431341) and Sanming Project of Medicine in Shenzhen (SZSM201801014).

\section{Authors' contributions}


Feiqi Zhu, PhD, MD: Designed the study, Analyzed the data. Interpreted the data. Took the lead in writing the manuscript. Kaiyu WU, MD: Analyzed the data. Interpreted the data. Wrote the manuscript. Chunyan Xu, MD, Xiaoping Tian, MD, Guozhen Qiu, MD, Qiwen Guo, MD, Xueya Meng, MD, and Chunchun Chen, MD: Collected the data. Accomplished MMSE assessments. Wei Liu, PhD, Zhiwei Guo,MD, and Jianjun Liu PhD: Designed the study. Collected the data. Haifei Chen, PhD,MD and Kangding Liu, PhD,MD: Analyzed the data. Interpreted the data. All the coauthors critically reviewed the manuscript and provided feedback. The authors read and approved the final manuscript.

\section{Acknowledgements}

We thank Prof. Peter Spencer from Oregon Health \& Science University, who shared his expertise on animal-based studies and English language use.

\section{References}

1. Gale SA, Acar D, Daffner KR (2018) Dementia. Am J Med 131, 1161-1169.

2. Hodson R (2018) Alzheimer's disease. Nature 559, S1.

3. Schuss P, Hadjiathanasiou A, Brandecker S, Güresir Á, Vatter H, Güresir E (2018) Elevated C-reactive protein and white blood cell count at admission predict functional outcome after non-aneurysmal subarachnoid hemorrhage. J Neurol 265, 2944-2948.

4. Dong X, Nao J, Shi J, Zheng D (2019) Predictive Value of Routine Peripheral Blood Biomarkers in Alzheimer's Disease. Front Aging Neurosci 11, 332.

5. Dagistan E, Cosgun Z (2019) Could hemogram parameters be predictors of dementia in elderly patients? Aging Male 22, 192-197.

6. Wang RT, Jin D, Li Y, Liang QC (2013) Decreased mean platelet volume and platelet distribution width are associated with mild cognitive impairment and Alzheimer's disease. J Psychiatr Res 47, 644-649.

7. Faux NG, Rembach A, Wiley J, Ellis KA, Ames D, Fowler CJ, Martins RN, Pertile KK, Rumble RL, Trounson B, Masters CL, Bush Al (2014) An anemia of Alzheimer's disease. Mol Psychiatry 19, 12271234.

8. Talib LL, Joaquim HP, Forlenza OV (2012) Platelet biomarkers in Alzheimer's disease. World J Psychiatry 2, 95-101.

9. Dos Santos GAA, Pardi PC (2020) Biomarkers in Alzheimer's disease: Evaluation of platelets, hemoglobin and vitamin B12. Dement Neuropsychol 14, 35-40.

10. Borroni B, Agosti C, Marcello E, Di Luca M, Padovani A (2010) Blood cell markers in Alzheimer Disease: Amyloid Precursor Protein form ratio in platelets. Exp Geronto/ 45, 53-56.

11. Liang QC, Jin D, Li Y, Wang RT (2014) Mean platelet volume and platelet distribution width in vascular dementia and Alzheimer's disease. Platelets 25, 433-438.

12. Deane R, Zlokovic BV (2007) Role of the blood-brain barrier in the pathogenesis of Alzheimer's disease. Curr Alzheimer Res 4, 191-197. 
13. Steubler V, Erdinger S, Back MK, Ludewig S, Fässler D, Richter M, Han K, Slomianka L, Amrein I, von Engelhardt J, Wolfer DP, Korte M, Müller UC (2021) Loss of all three APP family members during development impairs synaptic function and plasticity, disrupts learning, and causes an autism-like phenotype. Embo j, e107471.

14. Kamada Y, Hashimoto R, Yamamori H, Yasuda Y, Takehara T, Fujita Y, Hashimoto K, Miyoshi E (2016) Impact of plasma transaminase levels on the peripheral blood glutamate levels and memory functions in healthy subjects. BBA Clin 5, 101-107.

15. Neumann K, Farías G, Slachevsky A, Perez P, Maccioni RB (2011) Human platelets tau: a potential peripheral marker for Alzheimer's disease. J Alzheimers Dis 25, 103-109.

16. Farías G, Pérez P, Slachevsky A, Maccioni RB (2012) Platelet tau pattern correlates with cognitive status in Alzheimer's disease. J Alzheimers Dis 31, 65-69.

17. Korniluk A, Koper-Lenkiewicz OM, Kamińska J, Kemona H, Dymicka-Piekarska V (2019) Mean Platelet Volume (MPV): New Perspectives for an Old Marker in the Course and Prognosis of Inflammatory Conditions. Mediators Inflamm 2019, 9213074.

18. Chen SH, Bu XL, Jin WS, Shen LL, Wang J, Zhuang ZQ, Zhang T, Zeng F, Yao XQ, Zhou HD, Wang YJ (2017) Altered peripheral profile of blood cells in Alzheimer disease: A hospital-based case-control study. Medicine (Baltimore) 96, e6843.

19. Yesil Y, Kuyumcu ME, Cankurtaran M, Uz B, Kara A, Kilic MK, Halil M, Ulger Z, Yavuz BB, Haznedaroğlu IC, Ariogul S (2012) Increased mean platelet volume (MPV) indicating the vascular risk in Alzheimer's disease (AD). Arch Gerontol Geriatr 55, 257-260.

20. Sayed A, Bahbah El, Kamel S, Barreto GE, Ashraf GM, Elfil M (2020) The neutrophil-to-lymphocyte ratio in Alzheimer's disease: Current understanding and potential applications. J Neuroimmuno/349, 577398.

21. Shad KF, Aghazadeh Y, Ahmad S, Kress B (2013) Peripheral markers of Alzheimer's disease: surveillance of white blood cells. Synapse 67, 541-543.

22. Parker DC, Mielke MM, Yu Q, Rosenberg PB, Jain A, Lyketsos CG, Fedarko NS, Oh ES (2013) Plasma neopterin level as a marker of peripheral immune activation in amnestic mild cognitive impairment and Alzheimer's disease. Int J Geriatr Psychiatry 28, 149-154.

23. Leblhuber F, Walli J, Demel U, Tilz GP, Widner B, Fuchs D (1999) Increased serum neopterin concentrations in patients with Alzheimer's disease. Clin Chem Lab Med 37, 429-431.

24. Casal JA, Robles A, Tutor JC (2003) Serum markers of monocyte/macrophage activation in patients with Alzheimer's disease and other types of dementia. Clin Biochem 36, 553-556.

25. Pirttilä T, Mattinen S, Frey H (1992) The decrease of CD8-positive lymphocytes in Alzheimer's disease. J Neurol Sci 107, 160-165.

26. Pluta R, Ułamek-Kozioł M (2019) Lymphocytes, Platelets, Erythrocytes, and Exosomes as Possible Biomarkers for Alzheimer's Disease Clinical Diagnosis. Adv Exp Med Biol 1118, 71-82.

27. Richartz-Salzburger E, Batra A, Stransky E, Laske C, Köhler N, Bartels M, Buchkremer G, Schott K (2007) Altered lymphocyte distribution in Alzheimer's disease. J Psychiatr Res 41, 174-178. 
28. Zenaro E, Pietronigro E, Della Bianca V, Piacentino G, Marongiu L, Budui S, Turano E, Rossi B, Angiari S, Dusi S, Montresor A, Carlucci T, Nanì S, Tosadori G, Calciano L, Catalucci D, Berton G, Bonetti B, Constantin G (2015) Neutrophils promote Alzheimer's disease-like pathology and cognitive decline via LFA-1 integrin. Nat Med 21, 880-886.

29. Kuyumcu ME, Yesil Y, Oztürk ZA, Kizilarslanoğlu C, Etgül S, Halil M, Ulger Z, Cankurtaran M, Arıoğul S (2012) The evaluation of neutrophil-lymphocyte ratio in Alzheimer's disease. Dement Geriatr Cogn Disord 34, 69-74.

30. Rembach A, Watt AD, Wilson WJ, Rainey-Smith S, Ellis KA, Rowe CC, Villemagne VL, Macaulay SL, Bush Al, Martins RN, Ames D, Masters CL, Doecke JD (2014) An increased neutrophil-lymphocyte ratio in Alzheimer's disease is a function of age and is weakly correlated with neocortical amyloid accumulation. J Neuroimmunol 273, 65-71.

31. Shah RC, Wilson RS, Tang Y, Dong X, Murray A, Bennett DA (2009) Relation of hemoglobin to level of cognitive function in older persons. Neuroepidemiology 32, 40-46.

32. Andro M, Le Squere P, Estivin S, Gentric A (2013) Anaemia and cognitive performances in the elderly: a systematic review. Eur J Neurol 20, 1234-1240.

33. Zhang X, Zhou K, Wang R, Cui J, Lipton SA, Liao FF, Xu H, Zhang YW (2007) Hypoxia-inducible factor 1alpha (HIF-1alpha)-mediated hypoxia increases BACE1 expression and beta-amyloid generation. $J$ Biol Chem 282, 10873-10880.

34. Salminen A, Kauppinen A, Kaarniranta K (2017) Hypoxia/ischemia activate processing of Amyloid Precursor Protein: impact of vascular dysfunction in the pathogenesis of Alzheimer's disease. $J$ Neurochem 140, 536-549.

35. March-Diaz R, Lara-Ureña N, Romero-Molina C, Heras-Garvin A, Ortega-de San Luis C, Alvarez-Vergara MI, Sanchez-Garcia MA, Sanchez-Mejias E, Davila JC, Rosales-Nieves AE, Forja C, Navarro V, GomezArboledas A, Sanchez-Mico MV, Viehweger A, Gerpe A, Hodson EJ, Vizuete M, Bishop T, Serrano-Pozo A, Lopez-Barneo J, Berra E, Gutierrez A, Vitorica J, Pascual A (2021) Hypoxia compromises the mitochondrial metabolism of Alzheimer's disease microglia via HIF1. Nature Aging 1, 385-399.

36. Duce JA, Tsatsanis A, Cater MA, James SA, Robb E, Wikhe K, Leong SL, Perez K, Johanssen T, Greenough MA, Cho HH, Galatis D, Moir RD, Masters CL, McLean C, Tanzi RE, Cappai R, Barnham KJ, Ciccotosto GD, Rogers JT, Bush Al (2010) Iron-export ferroxidase activity of $\beta$-amyloid precursor protein is inhibited by zinc in Alzheimer's disease. Cell 142, 857-867.

37. Thirupathi A, Chang YZ (2019) Brain Iron Metabolism and CNS Diseases. Adv Exp Med Biol 1173, 119.

38. Park SE, Kim H, Lee J, Lee NK, Hwang JW, Yang JJ, Ye BS, Cho H, Kim HJ, Kim YJ, Jung NY, Son TO, Cho EB, Jang H, Jang EY, Hong CH, Lee JM, Kang M, Shin HY, Na DL, Seo SW (2016) Decreased hemoglobin levels, cerebral small-vessel disease, and cortical atrophy: among cognitively normal elderly women and men. Int Psychogeriatr 28, 147-156.

39. Brubaker WD, Crane A, Johansson JU, Yen K, Garfinkel K, Mastroeni D, Asok P, Bradt B, Sabbagh M, Wallace TL, Glavis-Bloom C, Tenner AJ, Rogers J (2017) Peripheral complement interactions with 
amyloid $\beta$ peptide: Erythrocyte clearance mechanisms. Alzheimers Dement 13, 1397-1409.

40. Chen Y, Lin C, Guo Z, Zhao S, Zhu Y, Huang F, Shui G, Lam SM, Pu J, Yan Y, Liu Z, Zhang B (2020) Altered Expression Profile of Phosphatidylinositols in Erythrocytes of Alzheimer's Disease and Amnestic Mild Cognitive Impairment Patients. J Alzheimers Dis 73, 811-818.

\section{Figures}

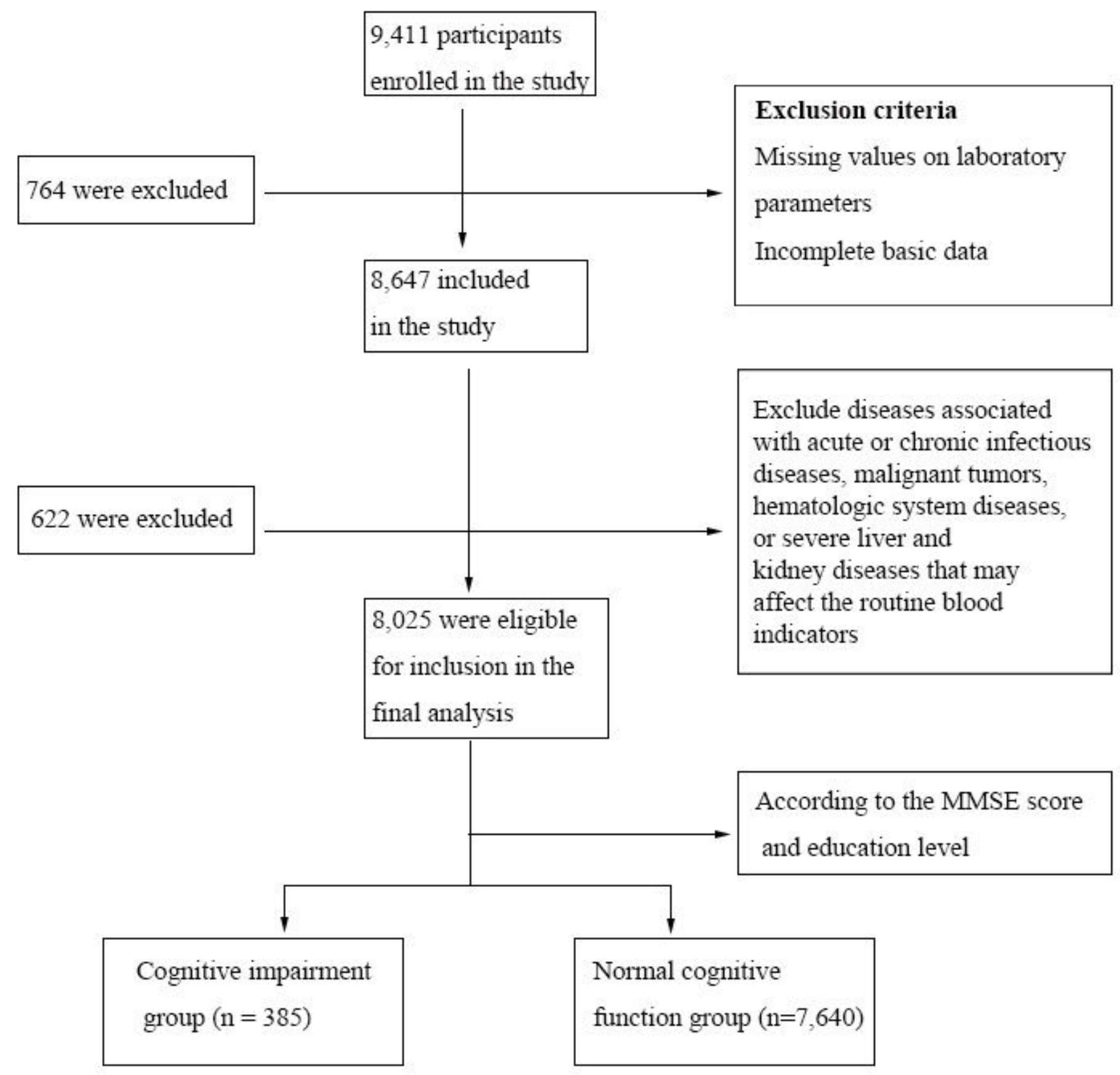

Figure 1

Flow diagram of this cross-sectional study 